\title{
Research and Application of Image De-noising in military
}

\author{
Feng $\mathrm{Yu}^{1,}$, , Feng Jiawei ${ }^{2,}$ a, Deng $\operatorname{Lin}^{3, \text { a }}$ \\ ${ }^{1}$ Training Department, Air Force Aviation University, changchun 130022, China \\ ${ }^{2}$ Aviation Training Base, Air Force Aviation University, changchun 130022, China \\ ${ }^{3}$ Computer Department, Air Force Aviation University, changchun 130022, China \\ aemail: gouna@sina.com
}

Keywords: Image De-noising; Wiener filter; BM3D; Military Image

\begin{abstract}
In order to hit the target accurately for military missile, its target image must be accurate. However, in practice the target image often encounter noise. Therefore, it is very important of image de-noising, and it has become a key step of the digital image pre-process. In this paper firstly we discuss the type of noise, and then we will introduction the de-noise principle of Wiener filtering and BM3D. At last the results show the effectiveness of method.
\end{abstract}

\section{Introduction}

In order to accurately hit the target for military missile, the target and the scene around it will be taken pictures by the television camera tube, and these will be transmitted to the guidance station through the transmitter. Data manipulation can see them on the screen, so it can be more intuitive manipulation missile to improve the accuracy. All images are transmitted through the form of digital image in the channel. The defender will often interfere with the channel using communication technology in order to reduce the accuracy of missile. The receiver receives the signal after filtering, and there is still an amount of noise in signal. When the signal noise ratio of the image is less than 1 , we cannot recognize the target in image, so it will makes missile guidance functions lost.

\section{The Type of Noise}

The image noise is that the image is suffered a random signal interference when it acquisition or transmission. It prevents us to accept their information for image. Many times the image noise is as a multi-dimensional stochastic process. So we can use the method of random process to describe image noise[1].

Classification by the relations of noise and signal

Additive noise: it is not relevant between additive noise and image signal strength. For operational amplifier and the image noise added in transmission, the image $g$ with this noise can be as the ideal image $\mathrm{f}$ with no noise adding the noise $\mathrm{n}$.

Multiplicative noise: it is relevant between additive noise and image signal strength. It often changes with the image signal varies. For the noise of scanned images, TV raster and film grain, these are multiplicative noise. The noise generated by carrying information for each pixel modulated by the information itself. In some cases, if the signal has small change, the same to noise.

Classification by the probability density function

Gaussian noise: In the spatial domain and frequency domain, Because of Gaussian noise (also called a normal noise) in mathematics tractability, this noise model is often used in practice.

Impulse noise: Bipolar impulse noises, also is known as salt and pepper noise, sometimes also called shot noise and spikes[2].

\section{The image de-noising algorithm}

There are many images de-noising algorithm. In this paper we focus on the algorithm of wiener 
filtering and BM3D. We will use the peak signal noise ratio (PSNR) to evaluate algorithm performance.

Wiener filtering: Wiener filter is a filter put forward by Wiener in the 1940s. It is an adaptive MMSE (Minimum Mean Square Error) filter. The method of wiener filter is a statistical method. The Optimal criteria it using is based on the correlation matrix of image and noise. It will adjust the output of filter by local variance of image and the bigger local variance, the stronger smoothing effect of the filter.

Wiener filter is an image recovery method with restraint. It integrates the characteristics both degraded image and image noise. Suppose the original image is $f(x, y)$ and De-noised image is $\hat{f}(x, y)$. Wiener filtering will make the least of mean square error between original image and de-noised image. As shown in Formula 1.

$$
E\left\{[f(x, y)-\hat{f}(x, y)]^{2}\right\}=\min
$$

In Equation 1, $E$ is mathematical expectation, so wiener filter is also known as the minimum mean Variance filter. Wiener filtering is based on minimizing the statistical criterion, and the results obtained from wiener filtering are optimal average sense. We can use Fourier transform to deduce the principles of wiener filtering.

$$
F(u, v)=H_{w}(u, v) G(u, v)=\frac{1}{H(u, v)} \cdot \frac{|H(u, v)|^{2}}{|H(u, v)|^{2}+\gamma \frac{p_{n}(u, v)}{p_{f}(u, v)}} \bullet G(u, v)
$$

Equation 2 restoration generic constraint expressions recovery, its transfer function is:

$$
H_{w}(u, v)=\frac{1}{H(u, v)} \cdot \frac{|H(u, v)|^{2}}{|H(u, v)|^{2}+\gamma \frac{p_{n}(u, v)}{p_{f}(u, v)}}
$$

BM3D:BM3D de-noising algorithm is currently the most common and performance image noise algorithm[3]. It uses the correlation for space and temporal in image to effective de-noise. BM3D de-noising algorithm is based on a three-dimensional block matching transform domain to filter. The image operation with the sliding window, the image is divided into several blocks, then search for each reference block in order to search similarity block. According to the degree of similarity of the block, the block matching will be combined to form a three-dimensional matrix. The matrix of data having high correlation, we can effectively reduce their relevance by three-dimensional unitary transformation. Meanwhile, by the transform domain coefficients filtering, the noise can be greatly reduced. Then the three-dimensional inverse transform can be estimated value of each block of similar. We get the finally de-noising image by estimating of all similar blocks to reconstruct weighting.

It has two steps for BM3D algorithm: it will get evaluated de-noising image using filtering methods with hard threshold and it will get the finally de-noising image using evaluated de-noising image and original image.

Detailed steps are as follows:

Step 1: Each element represented as an image block of $8 * 8$.

Step 2: Double orthogonal wavelet transform to each image block.

Step 3: Setting a threshold value.

Step 4: Calculate the distance difference between image blocks. We will take out the image block which distance difference is smaller than the threshold value. Re-assembled into the three-dimensional data, we will get 1-dimensional filtering for this three-dimensional data.

Step 5: We will get the finally result after inverse transform of the reconstructed image.

\section{Test Results}

The experiments divided into two part, these are we use wiener filtering algorithm to de-noise 
for images which have additive noise and multiplicative noise and we use BM3D algorithm to de-noise for images which have additive noise and multiplicative noise. In this paper the peak signal noise ratio (PSNR) will be used to evaluate algorithm performance especially for complex military texture image. The formulas of RMSE and PSNR will be given.

$$
\begin{aligned}
& R M S E=\sqrt{\frac{\sum_{x \in X}\left(u_{R}(x)-u_{D}(x)\right)^{2}}{|X|}} \\
& P S N R=20 \log _{10}\left(\frac{255}{R M S E}\right)
\end{aligned}
$$

Firstly, we will add noise to the original, then use wiener filtering and BM3D algorithms to de-noising. Last we calculate the PSNR based on the filtered results.

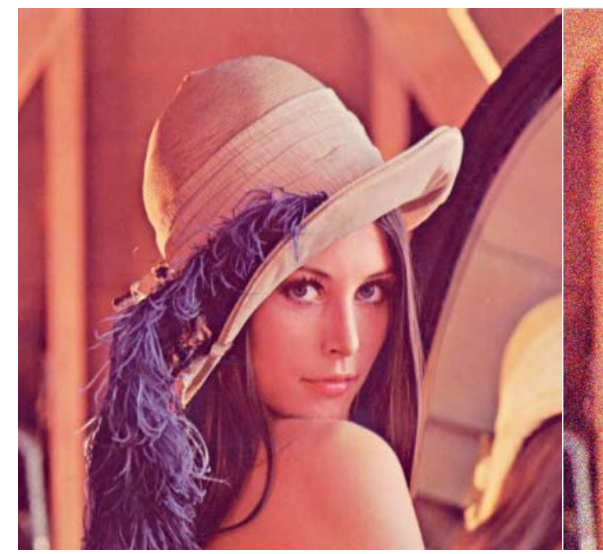

(a) Original Image

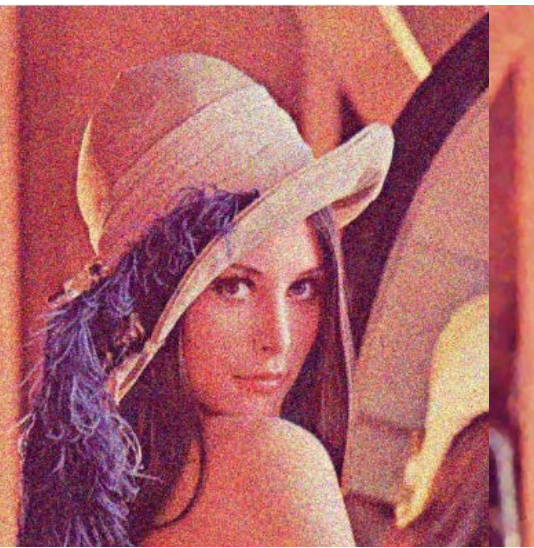

(b) Noisy Image

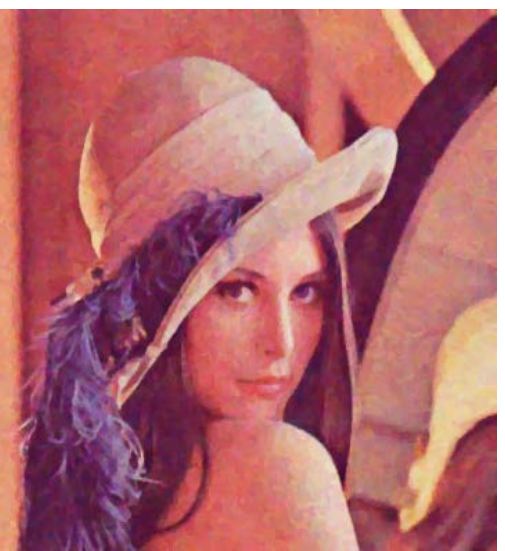

(c) De-noising Image

Fig.1 The wiener filter experimental results

For wiener filtering, the value of RMSE and PSNR are 37.996 and 16.536 before de-noising and after de-noising the value of RMSE and PSNR change to 9.079 and 28.969.

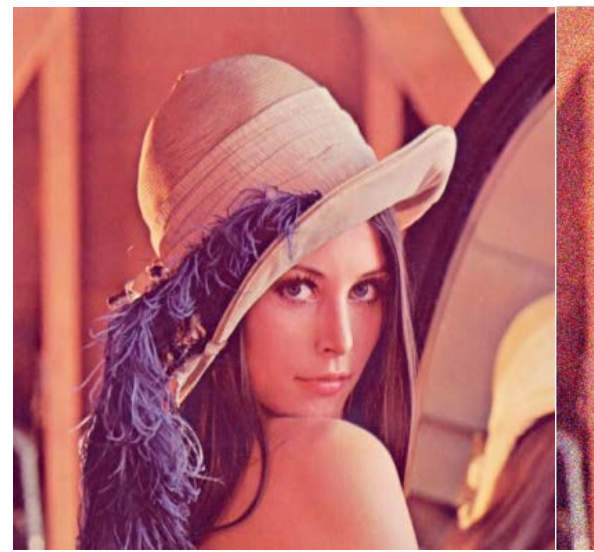

(a) Original Image

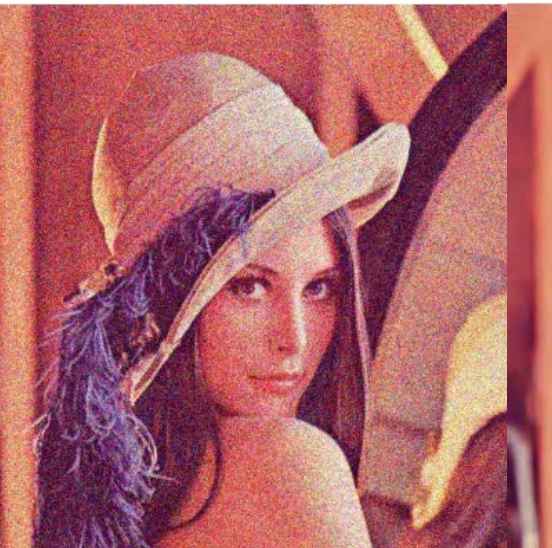

(b) Noisy Image

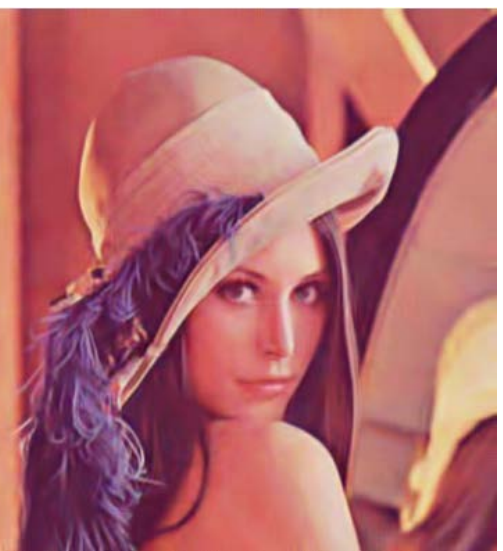

(c) De-noising Image

Fig. 2 The BM3D experimental results

For BM3D, the value of RMSE and PSNR are 42.426 and 14.795 before de-noising and after de-noising the value of RMSE and PSNR change to 10.441 and 29.755.From the results, Wiener filtering and BM3D algorithm can effectively de-noise in the image.

\section{Conclusion}

The image in reality almost has noise. When the noise is serious, it will affect the image segmentation, recognition and understanding especially for military image. Because the military image has more complex texture, the noise is likely to reduce image quality and impact of military activities. In this paper we focus on the common types of noise and the principles of de-noising 
algorithm. We also study the algorithm of wiener filtering and BM3D in military image. We get the applicability of the algorithm for different noise by comparing the experimental and data.

\section{References}

[1] Pok G, Liu J C, Nair A S. Selective Removal of Impulse Noise Based on Homogeneity Level Information[J]. IEEE Trans, On Image Processing, 2003,12(1):85-92.

[2] Kharrazi, M., Sencar, H. T., and Memon, N.: "Blind Source Came ra Identification”, Proc. ICIP’ 04 , Singapore, October 24-27, 2004.

[3] Kostadin Dabov, Alessandro Foi. Vladimir Katkovnik Image denoising with block-matching and 3D filtering 2006

[4] Kasparis T, Tzannes N S, Q Chen. Detail-preserving adaptive conditional median filters[J]. Electic. Image, 1992, 1 (14):356-364.

[5] Sawant A,Zeman H, Muratore D, etal. An adaptive median filter algorithm to remove impulse noise in X-ray and CT images and speckle in ultrasound images [J] proc. SPIE, 19991 3661(2):126 3-1274.

[6] Oppenheim A.V. and Schafer R.W. Digital Signal Processing. Prentice-Hall[J]. 1975:361-367.

[7] Popescu, A.C. and Farid H. Statistical Tools for Digital Forensic, in J. Fridrich: $6^{\text {th }}$ International Workshop on Information Hiding, LNCS vol. 3200, Springer-Verlag, Berlin-Heidelberg, New York, pp. 128-147, 2004.

[8] Farid, H. Exposing Digital Forgeries in Scientific Images. Proc. ACM Multimedia \& Security Workshop[C]. Geneva, Switzerland, pp. 29-36, 2006.

[9] Farid, H. and Lyu, S. Detecting Hidden Messages Using Higher-Order Statistics and Support Vector Machines, in F.A.P. Petitcolas (ed.): $5^{\text {th }}$ International Workshop on Information Hiding[C], LNCS vol. 2578, Springer-Verlag, Berlin-Heidelberg, New York, pp. 340-354, 2002.

[10] Holotyak, T., Fridrich, J., and Goljan, M.: "Estimation of Message Length Embedded Using Embedding, Proc. SPIE Electronic Imaging, Steganography, Security, an d Watermarking of Multimedia Contents VII, San Jose, California, January 16-20, 2005. 